\title{
Role of Disorder in the Size Scaling of Material Strength
}

\author{
Mikko J. Alava \\ Laboratory of Physics, Helsinki University of Technology, FIN-02015 HUT, Espoo, Finland \\ Phani K. V. V. Nukala \\ Computer Science and Mathematic Division, Oak Ridge National Laboratory, Oak Ridge, Tennessee 37831-6359, USA \\ Stefano Zapperi \\ INFM-CNR, S3, Dipartimento di Fisica, Università di Modena e Reggio Emilia, via Campi 213/A, I-41100, Modena, Italy \\ and ISI Foundation, Viale S. Severo 65, 10133 Torino, Italy \\ (Received 22 August 2007; published 5 February 2008)
}

\begin{abstract}
We study the sample-size dependence of the strength of disordered materials with a flaw, by numerical simulations of lattice models for fracture. We find a crossover between a regime controlled by the disorder and another controlled by stress concentrations, ruled by continuum fracture mechanics. The results are formulated in terms of a scaling law involving a statistical fracture process zone. Its existence and scaling properties are revealed only by sampling over many configurations of the disorder. The scaling law is in good agreement with experimental results obtained from notched paper samples.
\end{abstract}

DOI: 10.1103/PhysRevLett.100.055502

PACS numbers: 62.20.M-, 05.40.-a, 81.40.Np

The fracture strength of materials depends on various characteristic length scales of the specimen and represents a fundamental open problem of science and engineering. Probably the oldest scientific study of this issue was performed by Leonardo da Vinci, who measured the carrying capacity of metal wires of varying length [1]. The simple observation was that the longer the wire, the less weight it could sustain. This size effect can be understood considering that some form of disorder, such as dislocations, grains, or microcracks, is always present in materials. Hence, different parts of the sample should have a different strength depending of the local microstructure. In addition, the strength is not a self-averaging quantity since it is dominated by the weakest spot, where global failure is likely to initiate. Longer wires will, in general, contain more weak parts and are thus bound to fail earlier on average. While the physical mechanism behind this extreme-value based statistical size effect is clear, obtaining mathematical laws in realistic situations is still a formidable task [2].

In quasibrittle materials, such as concrete and many other composites, size effects are particularly complicated because of the significant damage accumulation preceding sample failure. The limitations of extreme-value statistics stem from long-ranged elastic interactions: cracks interact and it is not always obvious how to isolate the weakest spot. To overcome this problem, it is customary to consider a specimen containing a preexisting flaw, a notch. If the notch is sufficiently large, the stress enhancement around the crack tip will be sufficient to localize the failure. In this limit, disorder can be treated as a small perturbation [3] by defining a fracture process zone (FPZ) around the crack tip, where all the damage accumulation is confined. Several formulations to account for the size effects have been proposed in the literature [4-9]. These approaches are mainly based on the ad hoc extensions of linear elastic fracture mechanics (LEFM). This is a well established framework to understand cracks in homogeneous media, but it encounters fundamental problems when disorder is strong and homogenization methods are not applicable. This is particularly true for small notches, when failure is influenced by statistical effects and may, for instance, initiate far from the preexisting notch due to nucleated microcracks.

In LEFM the stability of a flaw against failure is given by the Griffith energy criterion, analogous to the classical theory of nucleation in first order phase transitions. Considering the elastic energy released by the crack and the interfacial energy gained in creating it, one can show that a crack of length $a_{0}$ becomes unstable for stresses larger than $\sigma_{c} \sim K_{c} / \sqrt{a_{0}}$, where the critical stress intensity factor $K_{c} \sim \sqrt{E G_{c}}$ is a function of the fracture toughness $G_{c}$ and the elastic modulus $E$ [10]. The Griffith argument has been generalized by Bazant to quasibrittle materials [4], postulating that when the FPZ is present, the crack length $a_{0}$ should increase by an additional length scale $\xi$, yielding

$$
\sigma_{c}=K_{c} / \sqrt{\xi+a_{0}} .
$$

Equation (1) incorporates two important effects: First, in the large notch limit $\xi / a_{0} \ll 0$ one should recover an expression that follows the LEFM scaling, in which the strength is inversely proportional to $1 / \sqrt{a_{0}}$. Second, for a vanishing external flaw size $a_{0} \rightarrow 0$, the average strength should still remain finite. In this limit, however, disorder cannot be considered as a small perturbation, and the assumptions underlying Eq. (1) are not valid. 
Here we investigate the role of the disorder in the failure of notched quasibrittle specimens, providing a microscopic justification and establishing the limits of validity of Eq. (1). We study the size scaling of strength by the extensive numerical simulations, a difficult task due to the different length scales involved and to the need of significant statistical averaging. We vary the disorder, which we model as a locally varying random failure threshold and show that it plays a crucial role in determining the size effect, influencing the fracture toughness $K_{c}$. Furthermore, the length scale $\xi$ naturally emerges from the simulations and can be shown to be directly related to the FPZ size. Finally, for notch sizes smaller than a critical length $a_{c}$, we observe a crossover to the inherent, samplesize-dependent strength of the unnotched sample. We present a scaling formula that incorporates all these effects and confirm its validity by comparing the simulations to experiments on notched paper samples.

To simulate a disordered elastic solid, we consider the simplest case where disorder and LEFM-like stress enhancements can be incorporated. We perform extensive simulations of the random fuse model (RFM) [11,12]. The RFM represents a quasibrittle failure process by an electrical analog composed of a network of fuses. We consider a triangular lattice of linear size $L$ with a central notch of length $a_{0}$. The fuses have unit conductance (which would correspond to $E=1$ in the elastic system) and random breaking thresholds $i_{c}$. These represent a locally varying fracture toughness or strength. The $i_{c}$ lie between 0 and 1 , with a cumulative distribution $P\left(i_{c}\right)=i_{c}^{1 / D}$, where $D$ represents a quantitative measure of disorder. The larger $D$ is, the stronger the disorder [13]. In the simulation, the burning of a fuse occurs irreversibly, whenever the electrical current in the fuse exceeds its threshold $i_{c}$. An external current is increased applying a voltage difference between the top and the bottom lattice bus bars and applying periodic boundary conditions along the other direction. Damage accumulates until a connected fracture path disconnects the network and one can define the strength $\sigma_{c}$ as the total peak current divided by the length of the bus bar. We also perform numerical simulations for the random spring model (RSM) [14], similar to the mesoscale models used routinely for concrete [15]. The RSM is similar to the RFM, but the fuses are replaced by elastic springs that break when their elongation reaches a random threshold. While the RSM represents more faithfully the elastic continuum, the statistical properties of the fracture process are analogous to those of the RFM [14].

The inset of Fig. 1 reports the strength, averaged over different configurations, with varying $a_{0}, D$, and $L$. The most instructive way of plotting is to consider the inverted square strength, $1 / \sigma_{c}^{2}$. Assuming Eq. (1), it is clear that $1 / \sigma_{c}^{2}$ should become a linear function of $a_{0}$ for large enough notches. Plotting the data in such a manner in Fig. 1 reveals four interesting features: (i) for $a_{0} \gg 1$,

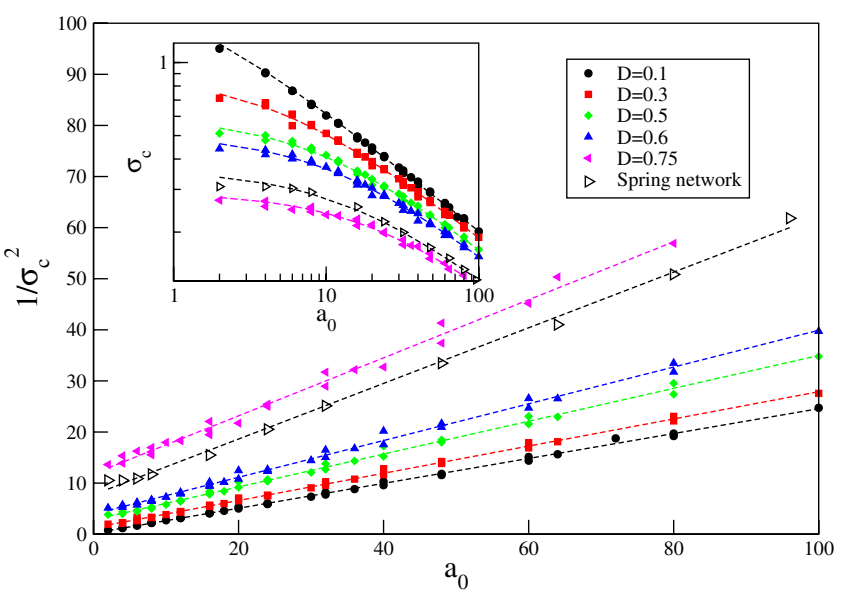

FIG. 1 (color online). Inset: The strength $\sigma_{c}$ as a function of the notch size $a_{0}$ for several disorders $D$. Results for different system sizes $L$ are plotted together. The main figure shows the scaling plot of the data according to Eq. (1).

the scaling of Eq. (1) is recovered asymptotically; (ii) the linear part of the data when extrapolated toward $a_{0}=0$ reveals a disorder-dependent intercept $\xi(D)$, that should be related to the size of the FPZ; (iii) the slope of the linear part of the data $\left[1 / K_{c}^{2}(D)\right]$ is disorder dependent, which implies a disorder-dependent fracture toughness $G_{c}(D)$; and finally, (iv) a careful observation (see Fig. 2) reveals that for small $a_{0}$ less than a critical crack size $a_{c}$, the strength scaling crosses over from a stress concentration dominated LEFM scaling [Eq. (1)] to a disorder-dominated scaling. In particular, the data presented in the inset of Fig. 2 indicate that the strength of the unnotched system (for $a_{0}=0$ ) is smaller than the LEFM limit $K_{c} / \sqrt{\xi}$ [where $1 / K_{c}^{2}$ is the slope and $\xi$ is the intercept of the lines in Fig. 1 based on Eq. (1)]. Hence, in order to extrapolate the typical sample-size-dependent strength from notched experiments

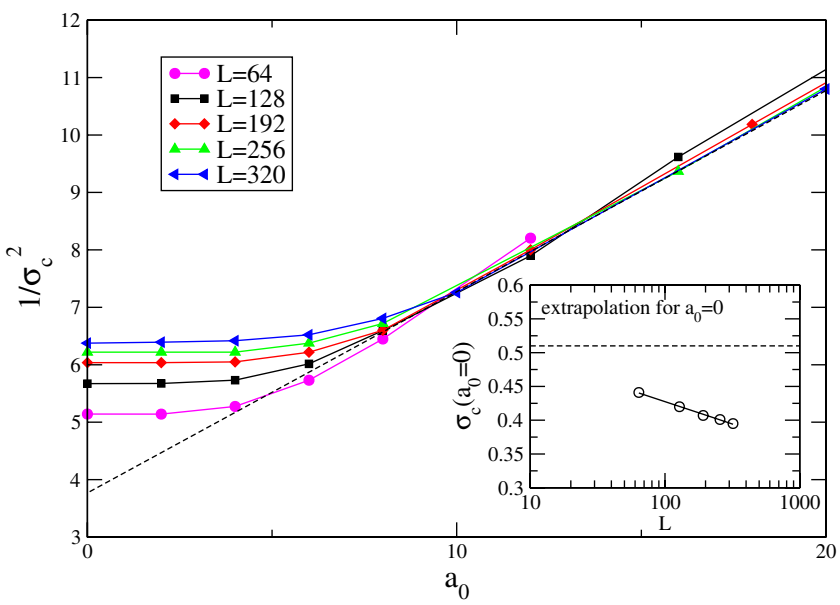

FIG. 2 (color online). A close-up of the strength data for $D=$ 0.6 and various $a_{0}$ and $L$. The inset compares fracture strength in unnotched samples with that predicted by Eq. (1) for $a_{0}=0$. 
it is necessary to know $a_{c}$. As can be seen, these features are also exhibited by RSM results.

The crossover at a critical crack size $a_{c}$ marks the important role of structural disorder or internal damage on the size effect and relates to the size effect in unnotched samples. For the RFM, the size effect without a notch has been shown by simulations and theoretical arguments to have a logarithmic dependence on the linear system size, $L$ $[12,16]$. For many engineering materials, one resorts to the Weibull theory [17] as an empirical starting point.

This crossover from LEFM dominated strength scaling to disorder-dominated strength scaling is illustrated in Fig. 3, where we compare the simulation data to experimental results on paper samples with varying center notch sizes. The paper data are from Ref. [18]. The two data sets presented are for strips of $L=15 \mathrm{~cm}$ cut from laboratorymade handsheets, of fine paper-type, with center defects of nominal sizes of $a_{0}=0.5,1,1.5,2$, and $2.5 \mathrm{~mm}$. Tensile tests were performed on 100 samples, in order to average the results. In Fig. 3, we observe clearly the presence of $a_{c}$, below which the notches have a negligible role on $\sigma_{c}$, and for crack sizes $a_{0}$ larger than $a_{c}$ fracture is ruled by LEFM. We have also fitted the simulation data to these experiments, by considering similar $a_{0} / L$ ratios and varying the $D$ to obtain reasonable agreement with the experimental data.

The observations from Figs. 1 and 2 can be summarized into a single scaling theory by noticing that there must be a scale $a_{c}$ above which the LEFM holds and $\sigma_{c}$ follows Eq. (1). For $a_{0} \ll a_{c}$ the strength scaling deviates significantly from Eq. (1) and saturates to a value that depends only on disorder and the sample size, $\sigma(L, D)$. This is the strength of the unnotched system of size $L$ and disorder $D$. The crossover from LEFM scaling [Eq. (1)] occurs at a notch size $a_{c}$ which can be obtained from Eq. (1) as

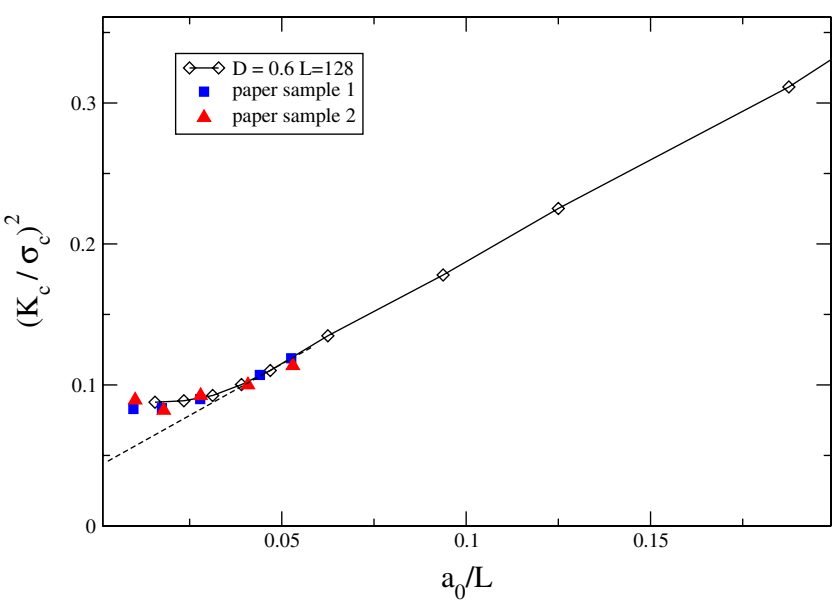

FIG. 3 (color online). Comparison of numerical results with the experimental data for two kinds of paper. The figure presents the size effect for small notch sizes using a RFM simulation of a system of size $L=128$ and a disorder of $D=0.6$.
$1 / \sigma(L, D)^{2} \simeq\left(a_{c}+\xi\right) / K_{c}^{2}$. It is possible to describe this crossover by the following scaling form, valid for all $a_{0}$ such that

$$
\frac{K_{c}^{2}}{\sigma_{c}^{2}}=\xi+a_{0} f\left(a_{c} / a_{0}\right),
$$

where the scaling function $f(y)$ fulfills the limits

$$
f(y) \simeq \begin{cases}1 & \text { if } y \ll 1, \\ y & \text { if } y \gg 1 .\end{cases}
$$

The length scale $a_{c} \simeq\left[K_{c}(D) / \sigma(L, D)\right]^{2}-\xi(D)$ corresponds to a crossover scale below which material strength is governed by disorder strength and system size. When the notch size exceeds this crossover length scale $\left(a_{0}>a_{c}\right)$, fracture is governed by LEFM. At fixed $L$, stronger disorder will increase $a_{c}$ since the strength of unnotched samples decays faster than the decrease in $K_{c}$. This is understandable since the disorder masks more efficiently the stress concentration due to the notch. Likewise, at fixed $D, a_{c}$ increases with increasing $L$.

Simulations of the RFM allow us to access the damage evolution prior to failure and can thus be used to visualize the development of the FPZ. As shown in Fig. 4, for a single realization of the disorder, at maximum stress we see only diffuse damage, without apparent localization so that the FPZ cannot be observed. When we average the damage over different configurations, however, a clear FPZ

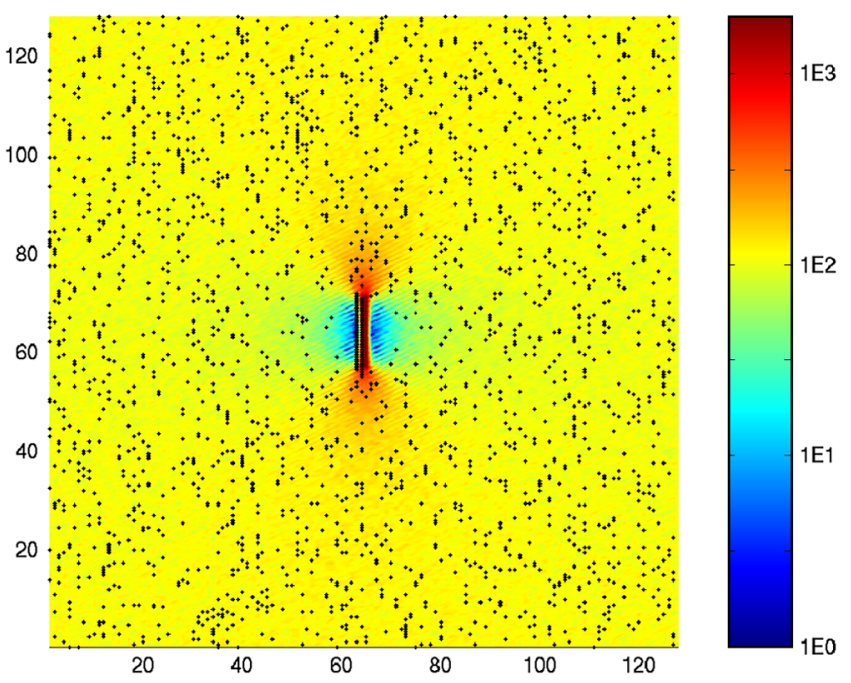

FIG. 4 (color online). The damage (fraction of failed elements or fuses) at maximum stress $\sigma_{c}$ for $L=128$ and $D=0.6$. The black markers illustrate the broken fuses in one single, randomly chosen sample whereas the color background represents the damage averaged over $N=2000$ (number of samples). The color code indicates the intensity of damage. A damage cloud that increases in density is clearly visible close to the initial notch. The figure demonstrates the screening of damage due to free crack faces. It is clear that in the disordered samples, FPZ is a statistical zone, visible only when damage profiles are averaged over many samples. 

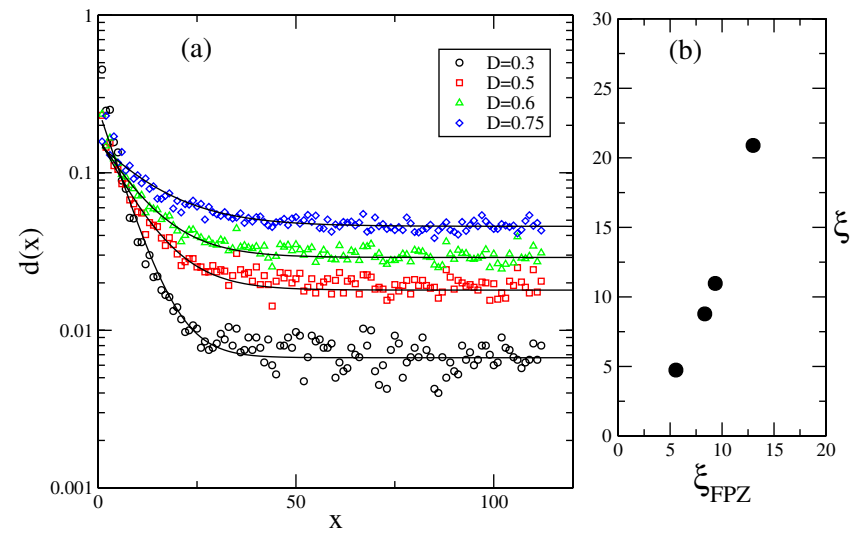

FIG. 5 (color online). (a) Damage profiles along the crack axis for various disorders $D$. Damage profiles follow an exponential decay on a uniform damage background, i.e., $d(x)=$ $A+B \exp \left(-x / \xi_{\mathrm{FPZ}}\right)$, where $A$ and $B$ are constants and $x$ is the distance from the crack tip along the crack axis. (b) $\xi$ vs $\xi_{\mathrm{FPZ}}$ for various disorders $D$.

emerges in front of the crack tip (see Fig. 4). Hence the FPZ should be considered a statistical concept, visible only when averaging over disorder, while the effect of the FPZ is nevertheless seen in the size effect $(\xi)$. To measure the FPZ size, we consider a projection of the average damage along the crack direction and obtain a profile that is decaying exponentially toward a homogeneous background value: $d(x)=A+B \exp \left(-x / \xi_{\text {FPZ }}\right)$ [see Fig. 5(a)]. We have analyzed the data for different values of $D$ and checked that the profiles do not depend on $a_{0}$ as long as this is not too far from $a_{c}$. The LEFM stress intensity factor would indicate a $1 / \sqrt{r}$-like divergence of the stress at the crack tip. It is evident that the observed exponential shape of the damage profile $d$ is in contrast to a $1 / \sqrt{r}$-like decay and should be naturally interpreted as a screening of the crack tip caused by the disorder. In Fig. 5(b), we plot the deduced fracture process size $\xi_{\mathrm{FPZ}}(D)$ against the intrinsic scale $\xi$ that one obtains from the fits of the strength data to the Eq. (1) and which also is an important part of the scaling theory presented in Eq. (2). It can be seen that these are linearly proportional indicating that $\xi$ is indeed a direct measure of the FPZ size. Notice that an exponential damage zone has indeed been measured in paper samples and the corresponding length scale was compared with the one obtained from Eq. (1) [19].

In conclusion, we have resorted to simulations of statistical fracture models to analyze the problem of the size effect in the failure of materials. For large notches, the simulations recover the expected scaling of LEFM [4] and allow us to relate the effective FPZ size $\xi$ to the actual average damage profiles. As the notch size is decreased we observe a crossover at a novel scale $a_{c}$ to a disorderdominated size-dependent regime that is not described by LEFM and is furthermore seen in experiments. All the regimes are summarized in a generalized scaling expression [Eq. (2)] for the strength of disordered media.

M. J. A. acknowledges the support of the Center of Excellence program of the Academy of Finland, discussions with Dr. R. Wathén, and the access to the data of Ref. [18]. M. J. A. and S.Z. gratefully thank the financial support of the European Commissions NEST Pathfinder programme TRIGS under contract No. NEST-2005-PATHCOM-043386. P. K. V. V.N. acknowledges support from Mathematical, Information and Computational Sciences Division, Office of Advanced Scientific Computing Research, U.S. Department of Energy under Contract No. DE-AC05-00OR22725 with UT-Battelle, LLC. P. K. V.V.N. also acknowledges the use of IBM BG/L resources made available to him at Argonne National Laboratory through INCITE. We are grateful to M. Gröhn and CSC, Finnish IT Center for Science, for assistance.

[1] L. da Vinci, I libri di Meccanica (Hoepli, Milan, Italy, 1940).

[2] E. J. Gumbel, Statistics of Extremes (Columbia University Press, New York, 2004).

[3] Z.P. Bazant and J. Planas, Fracture and Size Effect in Concrete and Other Quasibrittle Materials (CRC Press, Boca Raton, FL, 1997).

[4] Z.P. Bazant, Proc. Natl. Acad. Sci. U.S.A. 101, 13400 (2004).

[5] X. Hu and F. Wittmann, Mater. Struct. 25, 319 (1992).

[6] B. Karihaloo, Int. J. Fract. 95, 379 (1999).

[7] S. Morel, J. Schmittbuhl, E. Bouchaud, and G. Valentin, Phys. Rev. Lett. 85, 1678 (2000).

[8] S. Morel, E. Bouchaud, and G. Valentin, Phys. Rev. B 65, 104101 (2002).

[9] A. Carpinteri and N. Pugno, Nat. Mater. 4, 421 (2005).

[10] A. A. Griffith, Phil. Trans. R. Soc. A 221, 163 (1921).

[11] L. de Arcangelis, S. Redner, and H. J. Herrmann, J. Phys. (Paris), Lett. 46, 585 (1985).

[12] M. J. Alava, P. Nukala, and S. Zapperi, Adv. Phys. 55, 349 (2006).

[13] While this particular distribution has no deep physical justification, it is a convenient one to tune the disorder strength through a single parameter $(D)$. The results, however, should be independent of the particular distribution. Bond dilution disorder yields similar results.

[14] P. K. V. V. Nukala, S. Zapperi, and S. Simunovic, Phys. Rev. E 71, 066106 (2005).

[15] G. Lilliu and J. G. M. van Mier, Eng. Fract. Mech. 70, 927 (2003).

[16] P. M. Duxbury, P.D. Beale, and P. L. Leath, Phys. Rev. Lett. 57, 1052 (1986).

[17] W. Weibull, A Statistical Theory of the Strength of Materials (Generalstabens Litografiska Anstalts Förlag, Stockholm, 1939).

[18] R. Wathén, Lic. thesis, HUT, Espoo, Finland, 2003.

[19] H. Kettunen and K. Niskanen, J. Pulp Pap. Sci. 26, 35 (2000). 